\title{
Low-energy positron scattering from gas-phase benzene
}

\author{
Jan Franz ${ }^{1, a}$ and Małgorzata Franz ${ }^{2, b}$ \\ 1 Department of Theoretical Physics and Quantum Informatics, Faculty of Applied Physics and Mathematics, \\ Gdansk University of Technology, ul. Narutowicza 11/12, 80-233 Gdańsk, Poland \\ 2 Department of Physics of Electronic Phenomena, Faculty of Applied Physics and Mathematics, \\ Gdansk University of Technology, ul. Narutowicza 11/12, 80-233 Gdańsk, Poland
}

Received 31 January 2019 / Received in final form 5 June 2019

Published online 3 September 2019

(c) The Author(s) 2019. This article is published with open access at Springerlink.com

\begin{abstract}
In this paper we are presenting calculations of the elastic cross section of positrons with gasphase benzene for the energy range from $0.25 \mathrm{eV}$ to $9.0 \mathrm{eV}$. The calculations are done with the molecular R-matrix method for positron-scattering from poly-atomic molecules using a scaling factor to scale the electron-positron interaction. The scaling factor influences the position of the poles of the R-matrix. We adjust the scaling factor is such a way, that the position of the lowest pole is similar to the proposed binding energy of around $150 \mathrm{meV}$, given by [J.A. Young, C.M. Surko, Phys. Rev. Lett 99, 133201 (2007)]. Below the threshold for positronium formation, we calculate elastic cross section in good agreement with experimental cross sections. Above the threshold for positronium formation we use the difference between the experimental total cross section data and our computed elastic cross section to provide a first estimation of the cross section for positronium formation.
\end{abstract}

\section{Introduction}

Benzene is one of the simplest organic molecules. It is an ideal model compound to study interactions of positrons with biological matter. Such information is important for simulations of positron tracks in biological material $[1,2]$. These simulations require cross section data as an input. However, only a limited amount of experimental and theoretical cross section data are available for positron collisions with benzene. In a recent review Brunger et al. [3] point out that only total cross section data for positroncollisions with gas-phase benzene are available in the literature. The available experimental data show some disagreement between the experimental data sets from various groups. Occhigrossi and Gianturco [4] have done the only available theoretical study of this system.

An additional source of information on interactions of positrons with gas-phase benzene comes from the Surkogroup in San Diego, which has studied the annihilation of the colliding positrons depending on the collisional energy [5]. Young et al. [6-10] have used the model of vibrational Feshbach resonances to extract a binding energy of $150 \mathrm{meV}$. Recently Fedus [11] has analysed low-energy cross section data extract the scattering length with the modified effective range theory (MERT) [12]. As input for his MERT model he uses the scattering length obtained from the experimental binding energy of Young et al. [6]

\footnotetext{
${ }^{a}$ e-mail: jan.franz@pg.edu.pl

b e-mail: malgorzata.franz@pg.edu.pl
}

and obtains good agreement for the elastic scattering cross section at higher energies.

In this paper we present calculations of the elastic cross section of positrons with benzene molecules in the gasphase over the energy range from $0.25 \mathrm{eV}$ to $9.0 \mathrm{eV}$. For the calculations we are using the molecular R-matrix method for positron-scattering from poly-atomic molecules [13] with a semi-empirical scaling factor, which scales the electron-positron interaction [14]. The scaling factor scales the poles of the R-matrix. The method, its motivation and the computational details are described in Section 2.

In Section 3 we show the influence of the scaling factor on the poles of the R-matrix and on the elastic cross section. By using the scaling factor to move the lowest pole of the R-matrix to the value of the positron binding energy, we obtain an elastic cross section in good agreement with experimental data sets. We use difference between the experimental total cross section data of Karwasz et al. $[15,16]$ and our computed elastic cross section to estimate the cross section for positronium formation. The paper ends with conclusions in Section 4.

\section{Theoretical and computational methods}

\subsection{R-matrix method for positron-molecule scattering}

In the molecular R-matrix method for positron-scattering from poly-atomic molecules [13] the total wavefunction for $N$ electrons and one positron can be defined as 


$$
\begin{aligned}
& \Psi\left(\mathbf{x}_{1}, \mathbf{x}_{2}, \ldots, \mathbf{x}_{N} ; \mathbf{x}_{p} ; E\right) \\
& \quad=\sum_{k} A_{K}(E) \Psi_{K}\left(\mathbf{x}_{1}, \mathbf{x}_{2}, \ldots, \mathbf{x}_{N} ; \mathbf{x}_{p}\right) .
\end{aligned}
$$

Here $\mathbf{x}_{1} \ldots \mathbf{x}_{N}$ are the coordinates (both space and spin) of the electrons, $\mathbf{x}_{p}$ are the coordinates of the positron and $E$ is the collision energy. The coefficients $A_{K}(E)$ are energy-dependent and are obtained by propagating the Rmatrix outwards into the asymptotic region [17]. The functions $\Psi_{K}\left(\mathbf{x}_{1}, \mathbf{x}, \ldots, \mathbf{x}_{N} ; \mathbf{x}_{p}\right)$ are energy-independent and are eigenfunctions of the box-Hamiltonian $H_{\Omega}^{N+1}$

$$
H_{\Omega}^{N+1} \Psi_{K}\left(\mathbf{x}_{1}, \mathbf{x}_{2}, \ldots, \mathbf{x}_{N} ; \mathbf{x}_{p}\right)=E_{K} \Psi_{K}\left(\mathbf{x}_{1}, \mathbf{x}_{2}, \ldots, \mathbf{x}_{N} ; \mathbf{x}_{p}\right) .
$$

The eigenvalues $E_{K}$ are also called R-matrix poles. The box-Hamiltonian

$$
H_{\Omega}^{N+1}=H^{N+1}+L
$$

is confined to the sphere $\Omega$ and is the sum of the Hamiltonian $H^{N+1}$ for the $N+1$ particle system and the Bloch operator [18]

$$
L=\frac{1}{2} \delta\left(r_{p}-a\right)\left(\frac{d}{d r_{p}}-\frac{b-1}{r_{p}}\right) .
$$

Here $r_{p}$ is the radial distance of the positron from the scattering center, $a$ is the radius of the innermost R-matrix sphere and $b$ is an arbitrary constant. In this work we are choosing $b=0$. By adding the Bloch operator, the box-Hamiltonian $H_{\Omega}^{N+1}$ is hermitian and the Schrd̈inger equation is transformed into

$$
\begin{gathered}
\left(H_{\Omega}^{N+1}-E\right) \Psi\left(\mathbf{x}_{1}, \mathbf{x}_{2}, \ldots, \mathbf{x}_{N} ; \mathbf{x}_{p} ; E\right) \\
=L \Psi\left(\mathbf{x}_{1}, \mathbf{x}_{2}, \ldots, \mathbf{x}_{N} ; \mathbf{x}_{p} ; E\right)
\end{gathered}
$$

The R-matrix basis functions are constructed by the close-coupling expansion

$$
\begin{aligned}
\Psi_{K}\left(\mathbf{x}_{1}, \mathbf{x}_{2}, \ldots, \mathbf{x}_{N} ; \mathbf{x}_{p}\right)= & \sum_{A} \sum_{i} b_{A i}^{K} \Phi_{A}^{\mathrm{target}}\left(\mathbf{x}_{1}, \mathbf{x}_{2}, \ldots, \mathbf{x}_{N}\right) \phi_{i}\left(\mathbf{x}_{p}\right) \\
& +\sum_{B} \sum_{j} c_{B j}^{K} \Phi_{B j}^{N+1}\left(\mathbf{x}_{1}, \mathbf{x}_{2}, \ldots, \mathbf{x}_{N} ; \mathbf{x}_{p}\right) .
\end{aligned}
$$

The coefficients $b_{A i}^{K}$ and $c_{B j}^{K}$ are obtained variationally by diagonalizing the Hamiltonian $H_{\Omega}^{N+1}$. The first sum includes products of scattering functions $\phi_{i}\left(\mathbf{x}_{p}\right)$ of the positron and of the eigenfunctions $\Phi_{A}^{\text {target }}\left(\mathbf{x}_{1}, \mathbf{x}_{2}, \ldots, \mathbf{x}_{N}\right)$, of the Hamiltonian of the target molecule

$$
H_{\text {target }} \Phi_{A}^{\text {target }}\left(\mathbf{x}_{1}, \mathbf{x}_{2}, \ldots, \mathbf{x}_{N}\right)=E_{A}^{\text {target }} \Phi_{A}^{\text {target }}\left(\mathbf{x}_{1}, \mathbf{x}_{2}, \ldots, \mathbf{x}_{N}\right)
$$

with eigenvalue $E_{A}^{\text {target }}$. The second sum in the equation above runs over square-integrable functions for $N+1$ particles. Here the $N+1$ particles are $N$ electrons and one positron. These functions are confined to a sphere around the target molecule and their amplitude vanishes at the boundary.

At the boundary of the innermost R-matrix sphere the $N+1$ particle problem is projected onto an effective one-particle problem by integrating over the coordinates of the $N$ electrons. The $R$-matrix provides the boundary condition for the one-particle problem outside of the spherical box. More details can be found in e.g. Tennyson [17].

\subsection{R-matrix method with a semi-empirical scaling factor}

It is computationally very demanding to describe correlation between the impinging positron and the electrons without explicitly taking into account the positronelectron distance. In Franz et al. [14] we have introduced a semi-empirical scaling factor $f$, which scales the electronpositron attraction integrals. This approach has been successfully tested and applied to positron scattering from $\mathrm{CO}_{2}$ and $\mathrm{C}_{2} \mathrm{H}_{2}$ by Franz et al. [14,19].

The approach can be motivated by examination of the expression of the electron-positron correlation energy in second-order Møller-Plesset perturbation theory (see e.g. Takatsuka and Ten-no [20])

$$
E^{(2)}=\sum_{i a \bar{i} \bar{a}} \frac{(i a \mid \bar{i} \bar{a})}{\epsilon_{i}-\epsilon_{a}+\epsilon_{\bar{i}}-\epsilon_{\bar{a}}} .
$$

Here $\epsilon_{i}$ and $\epsilon_{a}$ denote energies of occupied and virtual electronic orbitals, respectively. $\epsilon_{\bar{i}}$ and $\epsilon_{\bar{a}}$ are the same for positrons. The positron-electron attraction integrals are given by

$$
(p q \mid \bar{r} \bar{s})=\int \phi_{p}\left(\mathbf{r}_{1}\right) \phi_{q}\left(\mathbf{r}_{1}\right)\left(-\frac{1}{\left|r_{1 \overline{1}}\right|}\right) \overline{\chi_{r}}\left(\mathbf{r}_{\overline{1}}\right) \overline{\chi_{s}}\left(\mathbf{r}_{\overline{1}}\right) d \mathbf{r}_{1} d \mathbf{r}_{\overline{1}}
$$

where $\phi_{p}\left(\mathbf{r}_{1}\right)$ and $\phi_{q}\left(\mathbf{r}_{1}\right)$ are electron orbitals, $\overline{\chi_{r}}\left(\mathbf{r}_{\overline{1}}\right)$ and $\overline{\chi_{s}}\left(\mathbf{r}_{\overline{1}}\right)$ are positron orbitals, and $\left|r_{1 \overline{1}}\right|=\left|\mathbf{r}_{1}-\mathbf{r}_{\overline{1}}\right|$ is the electron-positron distance. All orbital energies and orbital can be obtained by a generalized Hartree-Fock procedure for electron-positron systems [21].

However, in our implementation we are not using a generalized Hartree-Fock procudure and instead are using the same spatial orbitals for electrons and positrons. The firstorder contribution is given by

$$
E^{(1)}=\sum_{i \bar{i}}(i i \mid \overline{i i})
$$

The sum of the first- and second-order contribution can be rearranged as

$$
E^{(1+2)}=\sum_{i \bar{i}}\left((i i \mid \overline{i i})+(i i \mid \overline{i i})^{(2)}\right) .
$$

Here we have introduced a second-order correction $(i i \mid \overline{i i})^{(2)}$ to the electron-positron attraction integral $(i i \mid \overline{i i})$ as

$$
(i i \mid \overline{i i})^{(2)}=\sum_{i \bar{i}} \sum_{a \bar{a}} \frac{(i a \mid \bar{i} \bar{a})}{\epsilon_{i}-\epsilon_{a}+\epsilon_{\bar{i}}-\epsilon_{\bar{a}}} .
$$


For each electron-positron pair we can define a pairdependent scaling factor

$$
f_{i \bar{i}}=1+\frac{(i i \mid \overline{i i})^{(2)}}{(i i \mid \overline{i i})} .
$$

With this short-hand notation the sum of first and second-order electron-positron interaction energies can be written as

$$
E^{(1+2)}=\sum_{i \bar{i}} f_{i \bar{i}}(i i \mid \overline{i i}) .
$$

If we assume, that the scaling factor is the same for each electron-positron pair, we can replace the pair-dependent scaling factor by an averaged scaling factor $f$. The energy expression simplifies to

$$
E^{(1+2)} \approx f \sum_{i \bar{i}}(i i \mid \overline{i i})
$$

In our computer implementation we are scaling all positron-electron attraction integrals by the same factor

$$
(i j \mid \overline{i j})_{\mathrm{enh}}=f(i j \mid \overline{i j}) .
$$

Since we are using generalized configuration interaction wavefunctions, we encounter not only the integrals of the type $(i i \mid \overline{i i})$, but the more general type $(i j \mid \overline{i j})$. In this case the approximation used in equation (15) is less accurate.

The introduction of the scaling factor $f$ is moving the poles of the R-matrix. As can be seen in the next section, the R-matrix poles depend linearly from the scaling factor. In our calculations we are shifting the scaling factor $f$ in such a way, that the lowest R-matrix pole is close to the estimated positron-binding energy in benzene of $150 \mathrm{meV}$, as given by Gribakin et al. [5].

\subsection{Computational details}

The molecular geometry has been optimized with the program package Frisch et al. [22] using the B3LYP (Becke [23], three-parameter, Lee-Yang-Parr [24]) exchangecorrelation functional with the $6-311++\mathrm{G}^{* *}$ basis set [25].

For the scattering calculations we are using the modified UK molecular R-matrix codes of Gillan et al. [26], with the modifications described in Franz and Tennyson [13], Baluja et al. [27] and Franz et al. [14]. The target states are constructed from a complete active space configuration interaction (CAS-CI) wavefunction, where the complete active space is generated by distributing 4 electrons in 7 orbitals. All other electrons are kept frozen in the Hartree-Fock orbitals. The positron is allowed to occupy any of the occupied or virtual orbitals, which are used for electrons. In addition the positron can occupy continuum gaussian orbitals. These functions are located at centre of gravity of the molecule. The gaussian functions for the continuum basis function are constructed with the programs NUMCBAS and GTOBAS [30] following the procedure of Nestmann and Peyerimhoff [31]. The continuum basis set is contructed for $8 s, 7 p, 7 d, 6 f$ and $6 g$ functions

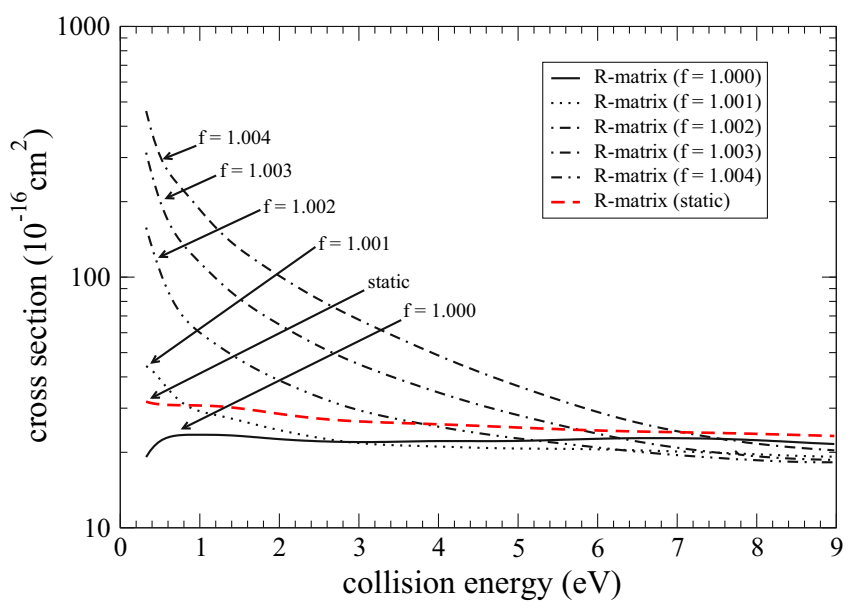

Fig. 1. Total elastic cross section for positron scattering from benzene for different values of the scaling factor $f$. For the static calculation (red, dashed line) a Hartree-Fock wavefunction is used for the electronic part. In all other calculations CAS-CI wavefunctions are employed. For more details see main text.

for a sphere with a radius of 14 bohr. In the actual scattering calculation the most-inner sphere has a radius of 13 bohr. In total this generates roughly 8000 configuration interaction functions for the construction of the total wavefunction. All calculations are done in the molecular point group $\mathrm{D}_{2 h}$. The propagation of the R-matrix into the asymptotic region is done with the $\mathrm{R}$-matrix propagator of Baluja et al. [32].

\section{Results and discussion}

\subsection{Influence of the scaling factor}

In Figure 1 the total elastic cross sections are shown for different values of the scaling factor $f$ using the CAS-CI model. For the value $f=1$ the scaling is switched off. In addition the cross section is shown for a static calculation, in which the electronic wavefunction of the target is represented by a Hartree-Fock wavefunction and and no other electronic functions are taken into account. The elastic cross section without scaling is a flat line and very similar to the static calculation with the Hartree-Fock target. This shows the difficulty to describe electron-positron correlation without taking into account the electron-positron distance explicitly. In our previous studies on positron collisions with the non-polar molecules $\mathrm{N}_{2}$ in Franz and Tennyson [13], $\mathrm{CO}_{2}$ in Franz et al. [14] and $\mathrm{C}_{2} \mathrm{H}_{2}$ in Franz et al. [19] we have obtained a similar picture.

A scaling factor larger than one is resulting in a markable increase of the elastic cross section at low collision energies. For larger collision energies we can see a small decrease in the cross section.

In Figure 2 the lowest R-matrix pole is shown as a function of the scaling factor $f$. The energy of the R-matrix pole is nearly a linear function of the scaling. In the following scattering calculations, which are presented in the next 


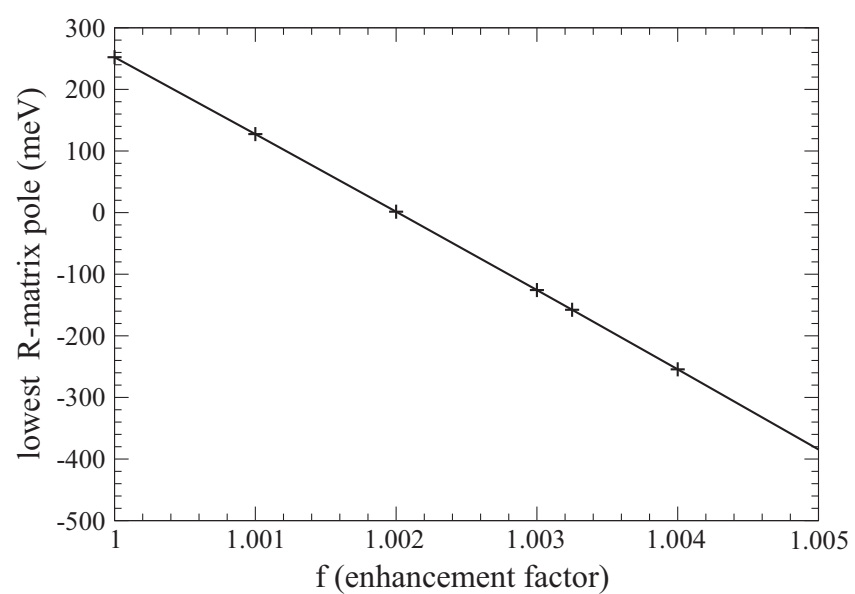

Fig. 2. Energy of the lowest R-matrix pole as a function of the scaling factor $f$. For more details see main text.

sub-section, we are using a scaling factor of $f=1.00325$. With this value of the scaling factor the lowest $\mathrm{R}$-matrix pole is at an energy of $157 \mathrm{meV}$. This value is very close to the estimated binding energy of $150 \mathrm{meV}$, obtained by the analysis of vibrational Feshbach resonances of energy resolved annihilitation spectra Young et al. [5-8,8-10].

\subsection{Comparison with results from the literature}

In Figure 3 our computed total elastic cross sections are shown for calculations without scaling $(f=1)$ and with the scaling factor of $(f=1.00325)$, which produces an R-matrix pole with a similar energy as the estimated positron-binding energy. Also shown are the calculations by Occhigrossi and Gianturco [4] and experimental data sets from Sueoka et al. [33], Makochekanwa et al. [34], Karwasz et al. [15,16] and Zecca et al. [35].

All experimental data sets are for total cross sections, including all inelastic channels, like rotational, vibrational and electronic excitation, positronium formation and ionisation. The experimental data sets differ from each other. Especially the data set by Makochekanwa et al. [34] deviates from the other data sets at low collision energies. The measurements of Makochekanwa et al. [34] are performed with the same scattering machine as those experiments of Sueoka [33] at Yamaguchi University. Brunger et al. [3] argue that the deviations could be a consequence of modifications in the spectrometer. The small differences between the data sets of Karwasz et al. $[15,16]$ and Zecca et al. [35] are mainly a result of an energy offset by $0.2 \mathrm{eV}$ in the collision energy.

The calculations by Occhigrossi and Gianturco [4] are done with potentials derived from density functional theory. The calculations are done for total elastic cross sections and do not include any inelastic channels. The authors used an asymptotic polarization potential to describe the longrange interaction between the positron and the molecule. Over the shown range of collision energies the calculations by Occhigrossi and Gianturco [4] are roughly 50 per-cent, as compared to the experimental data sets.

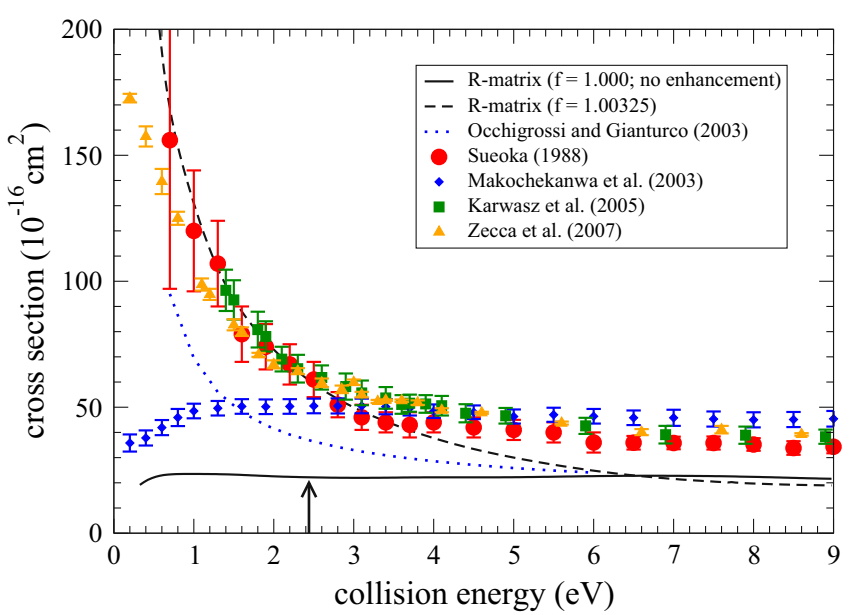

Fig. 3. Cross section for positron scattering from benzene. Also shown are calculations by Occhigrossi and Gianturco [4] and experimental data sets from Sueoka et al. [33], Makochekanwa et al. [34], Karwasz et al. [15,16] and Zecca et al. [35]. The arrow marks the threshold for positronium formation. For more details see main text.

The vertical ionization energy of benzene in the gasphase has been determined experimentally to be $9.240 \mathrm{eV}$ in Siegbahn's laboratory in Uppsala by Karlsson et al. [36]. Theoretically investigations by the group in Heidelberg have calculated a vertical ionization energy of $9.27 \mathrm{eV}$ using the equation-of-motion ionization potential coupled clusters singles and doubles (EOMIP-CCSD) method. Considering the experimental vertical ionization energy $\left(E_{\text {ion }}=9.24 \mathrm{eV}\right)$, the threshold for the formation of positronium is

$$
E_{\mathrm{Ps}}=E_{\text {ion }}-6.6 \mathrm{eV}=2.44 \mathrm{eV},
$$

where $6.8 \mathrm{eV}$ is the ground state energy of positronium [38]. This position is marked with an arrow in Figure 3.

For energies below the positronium formation threshold our calculations using the scaling factor are in good agreement with the experimental data sets of Sueoka [33] and Karwasz et al. $[15,16]$. At energies above the threshold for positronium formation, the differences between our calculations and the experimental data might be due opening of the channel for positronium formation. This channel is not included in our computed elastic cross sections, but is included in the experimental total cross sections.

\subsection{Estimation of the cross section for positronium formation}

The cross sections for inelastic rotional, vibrational and electronic excitation can be estimated to be of the order of a less than a few $10^{-16} \mathrm{~cm}^{2}$. Furthermore the cross section for ionization by positron impact is above the ionization threshold of $9.24 \mathrm{eV}$. Therefore we could use the difference between total cross section $\sigma_{\text {tot }}$ from the experimentat data set by Karwasz et al. $[15,16]$ and our computed elastic cross section $\sigma_{\mathrm{el}}$ to estimate the cross section for 
Table 1. Estimated cross sections for positronium formation. The collision energy is given in $\mathrm{eV}$. All cross section data are given in $10^{-16} \mathrm{~cm}^{2}$. The total cross section $\sigma_{\text {tot }}$ is taken from Karwasz et al. $[15,16]$. The elastic cross section $\sigma_{\mathrm{el}}$ is from this work using R-matrix theory with a scaling factor. The cross section for positronium formation $\sigma_{\mathrm{Ps}}$ is difference of the two cross sections.

\begin{tabular}{lrrr}
\hline Energy & $\sigma_{\text {tot }}$ & $\sigma_{\text {el }}$ & $\sigma_{\text {Ps }}$ \\
\hline 2.6 & 61.8 & 57.2 & 4.6 \\
2.9 & 58.2 & 51.6 & 6.6 \\
3.1 & 55.7 & 48.4 & 7.3 \\
3.4 & 53.7 & 44.4 & 9.3 \\
3.6 & 51.1 & 42.0 & 9.1 \\
3.7 & 51.6 & 40.8 & 10.8 \\
3.9 & 51.3 & 38.7 & 12.6 \\
4.1 & 50.6 & 36.8 & 13.8 \\
4.4 & 47.6 & 34.2 & 13.4 \\
4.9 & 46.6 & 30.6 & 16.0 \\
5.9 & 42.6 & 25.3 & 17.3 \\
6.9 & 39.1 & 21.8 & 17.3 \\
7.9 & 38.9 & 19.9 & 19.0 \\
8.9 & 38.3 & 19.0 & 19.3 \\
\hline
\end{tabular}

positronium formation

$$
\sigma_{\mathrm{Ps}} \approx \sigma_{\mathrm{tot}}-\sigma_{\mathrm{el}}
$$

The calculated values are given in Table 1 for the energy range $(2.6-8.9 \mathrm{eV})$. The cross section increases from $4.6 \times$ $10^{-16} \mathrm{~cm}^{2}$ at $2.6 \mathrm{eV}$ to $19.3 \times 10^{-16} \mathrm{~cm}^{2}$ at $8.9 \mathrm{eV}$.

It can be expected that the cross section for positronium formation is similar to the cross sections for positronium formation in organic molecules of similar size with similar ionization energies. Chiari et al. [39] have measured Positronium formation cross sections for positron collisions with tetrahydrofuran. They report an increase from $0.2 \times 10^{-16} \mathrm{~cm}^{2}$ at $2.6 \mathrm{eV}$ to $10.4 \times 10^{-16} \mathrm{~cm}^{2}$ at $9.0 \mathrm{eV}$. For positron collisions with pyrimidine Palihawadana et al. [40] report cross sections for positronium formation, which increase from $0.25 \times 10^{-16} \mathrm{~cm}^{2}$ at $2.0 \mathrm{eV}$ to $11.74 \times$ $10^{-16} \mathrm{~cm}^{2}$ at $9.0 \mathrm{eV}$. Our approximated cross section for positronium formation is about two times larger than for the two molecules, tetrahydrofuran and pyrimidine. This might be an indication, that our values are an overestimation.

\section{Present conclusions}

In this paper we have presented calculations of the elastic cross section of positrons with gas-phase benzene molecules for the energy range from $0.25 \mathrm{eV}$ to $9.0 \mathrm{eV}$. The calculations are done with the molecular R-matrix method for positron-scattering from poly-atomic molecules [13] using a scaling factor for scaling the electron-positron interaction [14]. By using the scaling factor we move the lowest pole of the R-matrix to the value of the proposed positron binding energy of Young et al. [6] and obtain an elastic cross section in good agreement with experimental data sets of Sueoka [33] and Karwasz et al. [15,16] for collision energy below the threshold for positronium formation. This can be interpreted as another indication for the existence of a positron bound-state in benzene with the binding energy of around $150 \mathrm{meV}$, as obtained by Young et al. [6]. Above the threshold for positronium formation we use difference between the experimental total cross section data and our computed elastic cross section to provide a first estimation of the cross section for positronium formation.

This work is supported by the grant 2014/15/D/ST2/02358 of the Polish National Science Center (Narodowe Centrum Nauki) and by computer grants from the computer centers WCSS (Wroclawskie Centrum Sieciowo-Superkomputerowe, Politechnika Wroclawska) and TASK (Trójmiejska Akademicka Sieć Komputerowa Gdańsk). The authors thank Gleb F. Gribakin (Queens University Belfast), Kamil Fedus and Grzegorz P. Karwasz (Nicolaus Copernicus University Torun) for fruitfull discussions.

\section{Author contribution statement}

Both authors contributed equally.

Open Access This is an open access article distributed under the terms of the Creative Commons Attribution License (http://creativecommons.org/licenses/by/4.0/), which permits unrestricted use, distribution, and reproduction in any medium, provided the original work is properly cited.

\section{References}

1. J. Baro, J. Sempau, J. Fernandez-Varea, F. Salvat, Nucl. Instrum. Methods Phys. Res. B 100, 31 (1995)

2. H. Aouchiche, C. Champion, D. Oubaziz, Radiat. Phys. Chem. 77, 107 (2008)

3. M.J. Brunger, S.J. Buckman, K. Ratnavelu, J. Phys. Chem. Ref. Data 46, 023102 (2017)

4. A. Occhigrossi, F.A. Gianturco, J. Phys. B: At., Mol. Opt. Phys. 36, 1383 (2003)

5. G.F. Gribakin, J.A. Young, C.M. Surko, Rev. Mod. Phys. 82, $2557(2010)$

6. J.A. Young, C.M. Surko, Phys. Rev. Lett 99, 133201 (2007)

7. J.A. Young, Energy-resolved annihilation studies: Vibrational feshbach resonances and positron-molecule bound states, Ph.D. thesis, University of California, San Diego, 2007

8. J.A. Young, C.M. Surko, Phys. Rev. A 77, 052704 (2008)

9. J.R. Danielson, J.A. Young, C.M. Surko, J. Phys. B: At., Mol. Opt. Phys. 42, 235203 (2009)

10. J.A. Young, C.M. Surko, Phys. Status Solidi C 6, 2265 (2009)

11. K. Fedus, J. Phys.: Conf. Ser. 618, 012029 (2015)

12. K. Fedus, G.P. Karwasz, Z. Idziaszek, Phys. Rev. A 88 012704 (2013) 
13. J. Tennyson, J. Franz, Low-energy electron and positron collisions with the R-matrix method, in Explicitly Correlated Wavefunctions, edited by E.A.G. Armour, J. Franz, J. Tennyson (CCP2, Daresbury, 2006), pp. 32-38

14. J. Franz, K.L. Baluja, R. Zhang, J. Tennyson, Nucl. Instrum. Methods Phys. Res. B 266, 419 (2008)

15. G.P. Karwasz, D. Pliszka, A. Zecca, R.S. Brusa, J. Phys. B: At., Mol. Opt. Phys. 38, 1 (2005)

16. G.P. Karwasz, A. Karbowski, Z. Idziaszek, R.S. Brusa, Nucl. Instrum. Methods Phys. Res. B 266, 471 (2008)

17. J. Tennyson, Phys. Rep. 491, 29 (2010)

18. C. Bloch, Nucl. Phys. 4, 503 (1957)

19. J. Franz, F.A. Gianturco, K.L. Baluja, J. Tennyson, R. Carey, R. Montuoro, R.R. Lucchese, T. Stoecklin, P. Nicholas, T.L. Gibson, Nucl. Instrum. Methods Phys. Res. B 266, 425 (2008)

20. A. Takatsuka, S. Ten-no, Bull. Korean Chem. Soc. 24, 859 (2003)

21. M. Müller, L.S. Cederbaum, Phys. Rev. A 42, 170 (1990)

22. M.J. Frisch, G.W. Trucks, H.B. Schlegel, G.E. Scuseria, M.A. Robb, J.R. Cheeseman, G. Scalmani, V. Barone, G.A. Petersson, H. Nakatsuji, X. Li, M. Caricato, A.V. Marenich, J. Bloino, B.G. Janesko, R. Gomperts, B. Mennucci, H.P. Hratchian, J.V. Ortiz, A.F. Izmaylov, J.L. Sonnenberg, D. Williams-Young, F. Ding, F. Lipparini, F. Egidi, J. Goings, B. Peng, A. Petrone, T. Henderson, D. Ranasinghe, V.G. Zakrzewski, J. Gao, N. Rega, G. Zheng, W. Liang, M. Hada, M. Ehara, K. Toyota, R. Fukuda, J. Hasegawa, M. Ishida, T. Nakajima, Y. Honda, O. Kitao, H. Nakai, T. Vreven, K. Throssell, J.A. Montgomery Jr., J.E. Peralta, F. Ogliaro, M.J. Bearpark, J.J. Heyd, E.N. Brothers, K.N. Kudin, V.N. Staroverov, T.A. Keith, R. Kobayashi, J. Normand, K. Raghavachari, A.P. Rendell, J.C. Burant, S.S. Iyengar, J. Tomasi, M. Cossi, J.M. Millam, M. Klene, C. Adamo, R. Cammi, J.W. Ochterski, R.L. Martin, K. Morokuma, O. Farkas, J.B. Foresman, D.J. Fox, Gaussian 16, Revision A.03 (Gaussian, Inc., Wallingford CT, 2016)

23. A.D. Becke, J. Chem. Phys. 98, 5648 (1993)
24. C. Lee, W. Yang, R.G. Parr, Phys. Rev. B 37, 785 (1988)

25. R. Krishnan, J.S. Binkley, R. Seeger, J.A. Pople, J. Chem. Phys. 72, 650 (1980)

26. C.J. Gillan, J. Tennyson, P.G. Burke, The UK molecular R-matrix scattering package: A computational perspective, in Computational Methods for Electronmolecule Collisions, edited by W. Huo, F.A. Gianturco (Plenum Press, New York, 1995), pp. 239-254

27. K.L. Baluja, R. Zhang, J. Franz, J. Tennyson, J. Phys. B: At., Mol. Opt. Phys. 40, 3515 (2007)

28. T.H. Dunning Jr., J. Chem. Phys. 53, 2823 (1970)

29. T.H. Dunning Jr., P.J. Hay, in Methods of Elelectronic Structure Theory, edited by H.F. Schaefer III (Plenum Press, New York, 1977), Vol. 2

30. A. Faure, J.D. Gorfinkiel, L.A. Morgan, J. Tennyson, Comput. Phys. Commun. 144, 224 (2002)

31. B.M. Nestmann, S.D. Peyerimhoff, J. Phys. B: At., Mol. Opt. Phys. 23, L773 (1990)

32. K.L. Baluja, P.G. Burke, L.A. Morgan, Comput. Phys. Commun. 27, 2993 (1982)

33. O. Sueoka, J. Phys. B: At., Mol. Opt. Phys. 21, L631 (1988)

34. C. Makochekanwa, O. Sueoka, M. Kimura, Phys. Rev. A 68, 32707 (2003)

35. A. Zecca, N. Moser, C. Perazzolli, A. Salemi, M.J. Brunger, Phys. Rev. A 76, 022708 (2007)

36. L. Karlsson, L. Mattsson, R. Jadrny, T. Bergmark, K. Siegbahn, Phys. Scr. 14, 230 (1976)

37. M. Döscher, H. Köppel, P.G. Szalay, J. Chem. Phys. 117, 2645 (2002)

38. M. Charlton, J.W. Humberston, Positron Physics (Cambridge University Press, Cambridge, 2001)

39. L. Chiari, E. Anderson, W. Tattersall, J.R. Machacek, P. Palihawadana, C. Makochekanwa, J.P. Sullivan, G. Garcia, F. Blanco, R.P. McEachran, M.J. Brunger, S.J. Buckman, J. Chem. Phys. 138, 074301 (2013)

40. P. Palihawadana, R. Boadle, L. Chiari, E.K. Anderson, J.R. Machacek, M.J. Brunger, S.J. Buckman, J.P. Sullivan, Phys. Rev. A 88, 012717 (2013) 